\title{
Neuro-cognitive Ramifications of Fasting and Feeding in Obese and Non-obese Cases
}

\author{
Seyed-Ali Mostafavi ${ }^{1}$, Ali Khaleghi ${ }^{1}$, Safa Rafiei Vand ${ }^{2}$, Seyyed Salman Alavi ${ }^{1}$, Mohammad Reza Mohammadi ${ }^{1}$ \\ ${ }^{1}$ Psychiatry and Psychology Research Center, Tehran University of Medical Sciences, ${ }^{2}$ Faculty of Biomedical Engineering, Amirkabir University \\ of Technology, Tehran, Iran
}

\begin{abstract}
Preliminary studies have claimed that short term fasting would negatively affect school performance and cognition. In contrast some other studies have reported not important decline in cognition and executive function as a result of fasting. Also limited attention was generally devoted to dietetic regimens, nutritional status and body weight. Yet neuroscience and neuro-cognitive aspects of acute hunger on the electroencephalogram and differences between obese and non-obese cases is not well understood. Hence, we decided to design and perform a case study in a more controlled situation similar to reality. Therefore, we performed several examinations including subjective tests (for eating status) and objective tests (cognitive tests such as Stroop effect and Sternberg search and electroencephalogram measures such as steady-state visual evoked potential and auditory steady-state responses) for an obese and a non-obese academic case before and after a simple breakfast. The results showed that the breakfast effects on the neuro-cognitive functions depend on either obesity status, nutritional status of the case or the type of cognitive task (visual or auditory). This paper would open a new insight to answer some important questions about the neuro-cognitive implications of fasting and feeding in obese and non-obese human cases.
\end{abstract}

KEY WORDS: Electroencephalography; Fasting and Feeding; Neuro-cognition; Obesity.

\section{INTRODUCTION}

Preliminary studies have claimed that short term fasting would negatively affect school performance and cognition. In contrast some other studies have reported not important decline in cognition and executive function as a result of fasting. ${ }^{1)}$ Most of these studies had confounded by moderators or confounders of cognitive and academic performance such as motivation, facilities, quality of teaching, socioeconomic status, parents' educational level, and attitudes toward school. ${ }^{2)}$ Furthermore, most of abovementioned studies were poorly controlled used breakfasts with different composition and energies, and used subjects of different ages making it very difficult to

Received: April 20, 2017 / Revised: June 29, 2017

Accepted: July 14, 2017

Address for correspondence: Ali Khaleghi, PhD

Psychiatry and Psychology Research Center, Tehran University of Medical Sciences, South Kargar Ave., Roozbeh Hospital,

Tehran, 1333795914, Iran

Tel: +98-9163464494, Fax: +98-21-55421959

E-mail: a-khaleghi@razi.tums.ac.ir

ORCID: https://orcid.org/0000-0002-9035-7075 construct a very strong conclusion. ${ }^{3)}$ Also, most of these kinds of studies were performed in school aged children. Some researchers have criticized that academic achievement in breakfast eaters may be confounded by higher socioeconomic status; and higher rate of attendance in school for breakfast. Moreover, cognitive functions will decline in some psychopathological conditions. ${ }^{4)}$

Yet neuroscience and neuro-cognitive aspects of acute hunger and characteristics of electroencephalogram (EEG) and differences between obese and non-obese cases is not well understood. To eliminate the abovementioned confounders we decided to design and perform a case study in a more controlled situation similar to reality.

\section{MATERIALS AND METHODS}

Two cases one obese and the other non-obese, invited to participate in this study. The protocol of the study was described to them and they were gave consent form and signed it. They were entered into the analysis including body composition analysis, cognitive, and EEG assess-

(ㄷ) This is an Open-Access article distributed under the terms of the Creative Commons Attribution Non-Commercial License (http://creativecommons.org/licenses/by-nc/4.0) which permits unrestricted non-commercial use, distribution, and reproduction in any medium, provided the original work is properly cited. 
ments each performed in two consecutive days. They were asked to arrive in fasting state in morning to perform the assessments in standard situation. Sternberg search and Stroop tests were completed by two subjects before and after the breakfast to assess the cognitive function and to determine the reaction power in obese and non-obese cases. Furthermore both subjects completed the validated eating behavior questionnaires including visual analogue scale for appetite, visual analogue scale for restrained eating, Food Craving Questionnaire (FCQ), Three Factor Eating Questionnaire, Compulsive Eating Scale (CES) as well as Beck depression inventory. The semi structured interview was performed by a psychiatrist based on Diagnostic and Statistical Manual of Mental Disorders, 5th edition (DSM-5) interview. Subjects also were asked to record everything they eat for three days (two working days and one weekend).

\section{Body Composition Analysis}

We used Omron HBF-500 BIA (Omron Co., Kyoto, Japan) device which involve eight electrodes, a tetrapolar electrodes in footpads and another 4 set of electrodes in handle. The subjects stood on metal footpads in bare feet and grasped a pair of electrodes fixed on a handle with arms extended in front of the chest. This instrument assesses total body fat, visceral fat, lean body mass, and basal metabolic rate as well as body weight and body mass index. The clinical validity of this instrument in measuring body composition is already approved in comparison with dual-energy $\mathrm{X}$-ray absorptiometry and magnetic resonance imaging.

\section{Nutritional Assessments Tools}

\section{Three-Factor Eating Questionnaire-R18}

This test is a widely used tool in surveys of eating behavior. The psychological characteristics, and validity and reliability of the questionnaire had been evaluated and approved previously. It has good internal consistency $(\mathrm{a}=0.73)$, and test-retest reliability $(\mathrm{r}=0.87)$. This questionnaire is able to distinguish among different eating patterns in a general population. ${ }^{6}$

\section{Compulsive Eating Scale (CES)}

This instrument was first created by Kagan and Squires. It is an 8-item self-report instrument made to measure the severity of binge eating disorder. This test has been validated in Iranian obese individuals. Factor analysis of this instrument showed 2 factors: eating because of negative feelings and overeating. The internal consistency of the CES was 0.85. ${ }^{7)}$

\section{Food Craving Questionnaire (FCQ)}

The clinical validity of this instrument was approved in patients with eating disorder. The internal consistency and reliability indexes of the tool ranged from moderate to excellent. This scale could predict the symptoms of eating disorder. The factor analysis, and the reliability and validity of the Persian version of FCQ are under study by the current team. ${ }^{8)}$

\section{Beck depression inventory}

It is a 21 -item self-report questionnaire that its reliability and validity is accepted worldwide and assesses symptoms of depression in adults. Its Persian version has a high internal consistency (Cronbach's alfa, 0.87) and acceptable test-retest reliability $(r=0.74) .^{9)}$

\section{Appetite visual analogue scale}

This rating scale is widely used in appetite research. Parker et al. ${ }^{10)}$ tried to assess the validity of the visual analogue scale and reported a significant correlation between food intake and this tool.

\section{EEG Recording and Analysis}

Recording of EEG signal was done from 16 active electrodes using USBamp biosignal amplifier (g.tec, Schiedlberg, Austria). Electrode locations according to 10 to 20 standards were chosen on Fp1, Fp2, F3, F4, F7, F8, Fz, C3, C4, Cz, T7, T8, Cp5, Cp6, O1 and O2. Reference electrode was placed on the right ear. Sampling rate was 512 $\mathrm{Hz}$ and cut-off frequency of the amplifier's electronic low pass and high pass filters were adjusted on 0.1 and $60 \mathrm{~Hz}$ respectively. ${ }^{11)}$ To avoid saccadic and other eye movements, a white cross in black background was shown to subjects in a monitor and was asked them to gaze on it. Each recording session was at least 132 seconds (40 trial $\times 1.8$ seconds+1.5 seconds interval between each trial).

To extract steady state response, a moving average window with the length equal to five period of the stimulation (333 ms for visual stimulation and $125 \mathrm{~ms}$ for auditory) was moved on each trial with 4-period overlap. Finally, 
5-period time segment of all trials were averaged. Lock-in analyzer system was utilized for calculation of amplitude and phase of the response. ${ }^{12)}$

\section{Cognitive Assessment}

\section{Sternberg search}

Many researchers of memory routinely use the computer based "Sternberg search" for assessing the short-term memory. The process of this test on coglab as described by @ Cengage Learning is as follow: "a memory set consisting of 1,3 , or 5 digits will appear on the screen for 1.2, 3.6, or 6 seconds, respectively. The client is asked to study the numbers and keep them into the memory. The memory set will disappear and then a probe item will appear 1 to 3 seconds later. The client task is to determine as quickly as possible whether the probe item was in the list of just presented numbers (respond present) or was not in the list (respond absent). If the the client make a mistake (e.g., say the item was present when it was not), the trial will be repeated (with different numbers) later in the experiment. In this way, only response times for trials in which the client was correct are used". ${ }^{13)}$

\section{Stroop tests}

The Stroop test is one of the best known tools to assess cognition including selective attention. It measures the ability of person to react to a definite environmental stimulus whilst overlook others. John Ridley Stroop was the first who reported this effect in his $\mathrm{PhD}$ thesis published in 1935, commonly known as "Stroop effect". ${ }^{14)}$

\section{Breakfast Characteristics}

We examined the effects of the same mixed breakfast meal for participants including cheese, bread, walnut, tea and sugar (total breakfast energy: $350 \mathrm{kcal})$. Both cases were habitual breakfast eaters. The amount of energy and breakfast composition was equal in both cases.

\section{RESULTS AND DISCUSSION}

Both cases were the same in regards with socio-economic status, age, and mood but different in body analysis characteristics, subjective tests for appetite, restrained eating, eating problems, and usual dietary intake (before participating) (Table 1). Both cases had similar sedentary physical activity in the last week before the examinations.

As shown in Figure 1, the amplitude and phase of steady-state visual evoked potential (SSVEP) responses are reduced in obese case after eating breakfast that indicating increased functional activity and increased speed of visual information processing, respectively, in the fed state. ${ }^{15)}$ Whereas for non-obese subject, the phase of SSVEP responses are only decreased and the amplitude remains almost unchanged after eating breakfast. This suggests that despite the relatively stable level of activity, speed of visual information processing is increased in the occipital region for non-obese subject. Results obtained from auditory steady-state responses (ASSR) characteristics are different for both subjects. For the obese case in the fed state, the amplitude of ASSR is decreased but its phase is raised compared to the fasting state, suggesting increased functional activity and decreased speed of auditory information processing in the temporal region. For the non-obese case, both amplitude and phase of ASSR are increased after eating breakfast, suggesting reduced activity and speed of auditory information processing in the temporal region.

Table 1. Basic characteristics mean dietary intake and body analysis of two cases

\begin{tabular}{lcc}
\hline \multicolumn{1}{c}{ Characteristic } & $\begin{array}{c}\text { Obese } \\
\text { case }\end{array}$ & $\begin{array}{c}\text { Non-obese } \\
\text { case }\end{array}$ \\
\hline Age $(\mathrm{yr})$ & 27 & 26 \\
Weight $(\mathrm{kg})$ & 109.3 & 67.8 \\
Body mass index $\left(\mathrm{kg} / \mathrm{m}^{2}\right)$ & 35.7 & 21.4 \\
Waist circumference $(\mathrm{cm})$ & 121 & 85 \\
Hip circumference $(\mathrm{cm})$ & 116 & 98 \\
Total fat rate $(\%)$ & 39 & 18.9 \\
Visceral fat $(\%)$ & 16 & 4 \\
Muscle rate $(\%)$ & 29 & 39.8 \\
Basal metabolic rate (kcal) & 2,153 & 1,599 \\
Three-Factor Eating Questionnaire & 42 & 29 \\
Visual analogue scale for restrained eating & 6 & 4 \\
Beck depression inventory & 2 & 4 \\
Compulsive Dating Scale & 19 & 10 \\
Visual analogue scale for appetite & 14 & 9 \\
Food Craving Questionnaire & 42 & 18 \\
Dietary intake & & \\
Mean daily total energy intake (kcal) & 2,469 & 2,193 \\
Mean protein intake (g) & 76.6 & 76.3 \\
Mean fat intake (g) & 124.5 & 87.4 \\
Mean carbohydrate intake (g) & 274 & 283 \\
Mean iron intake (mg) & 14.6 & 12.7 \\
Mean zinc intake (mg) & 8.9 & 9.6 \\
Mean sugar intake (g) & 58.6 & 58.1 \\
\hline
\end{tabular}




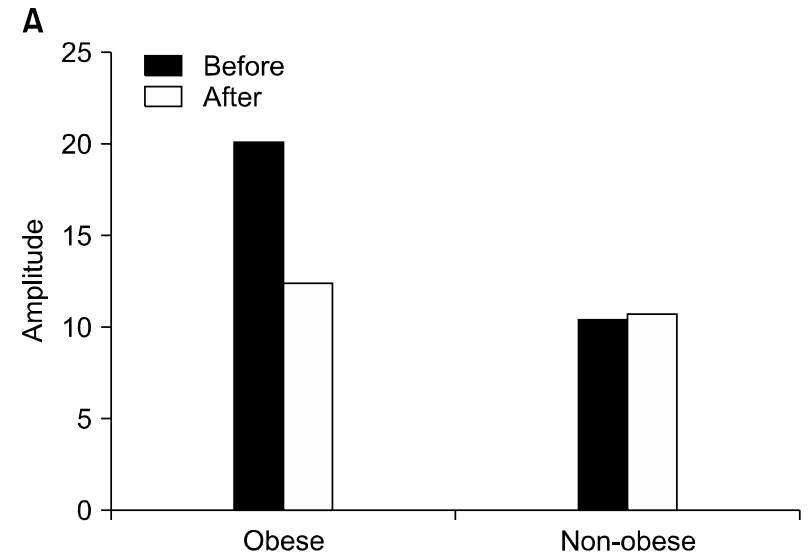

B

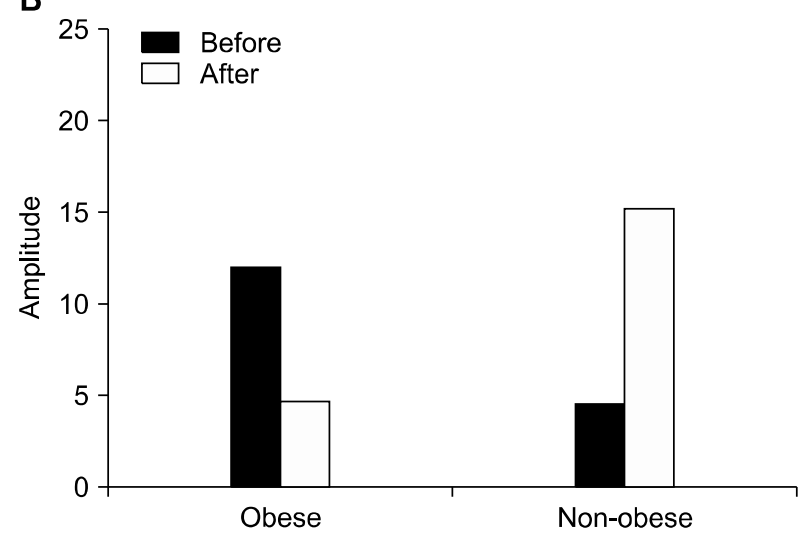

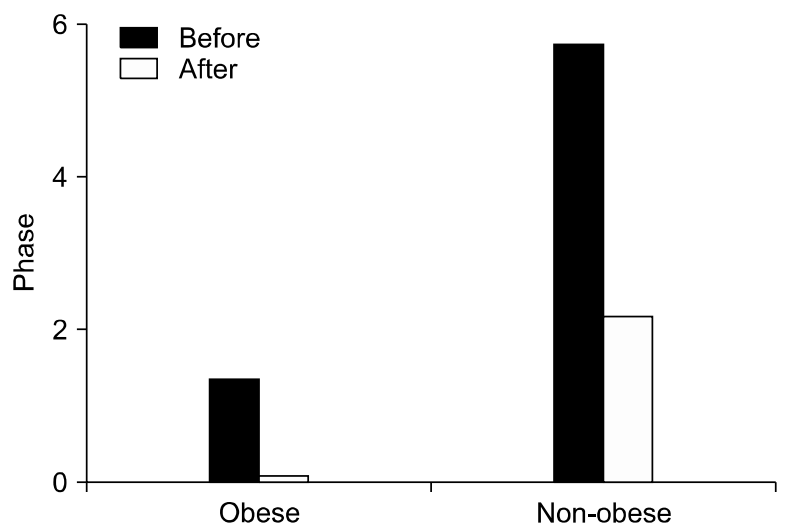

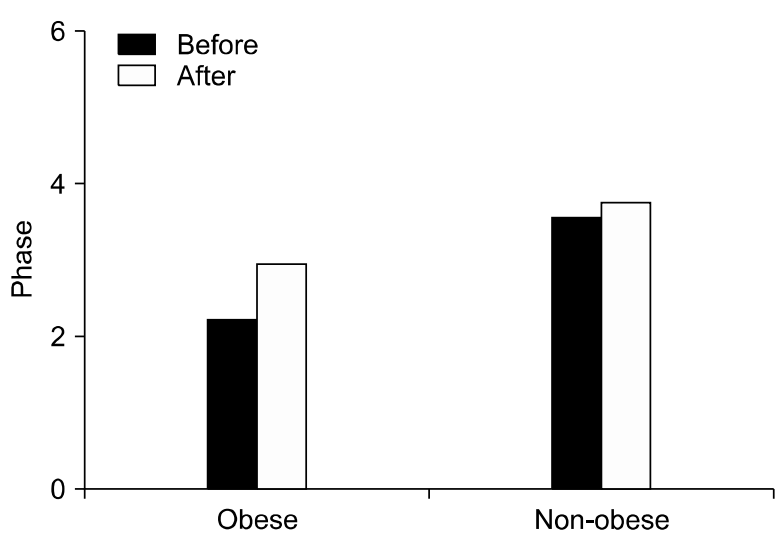

Fig. 1. Amplitude and phase of steady-state visual evoked potential responses from occipital region (A) and auditory steady-state responses from bilateral temporal region (B) for obese and non-obese cases, before and after eating breakfast.
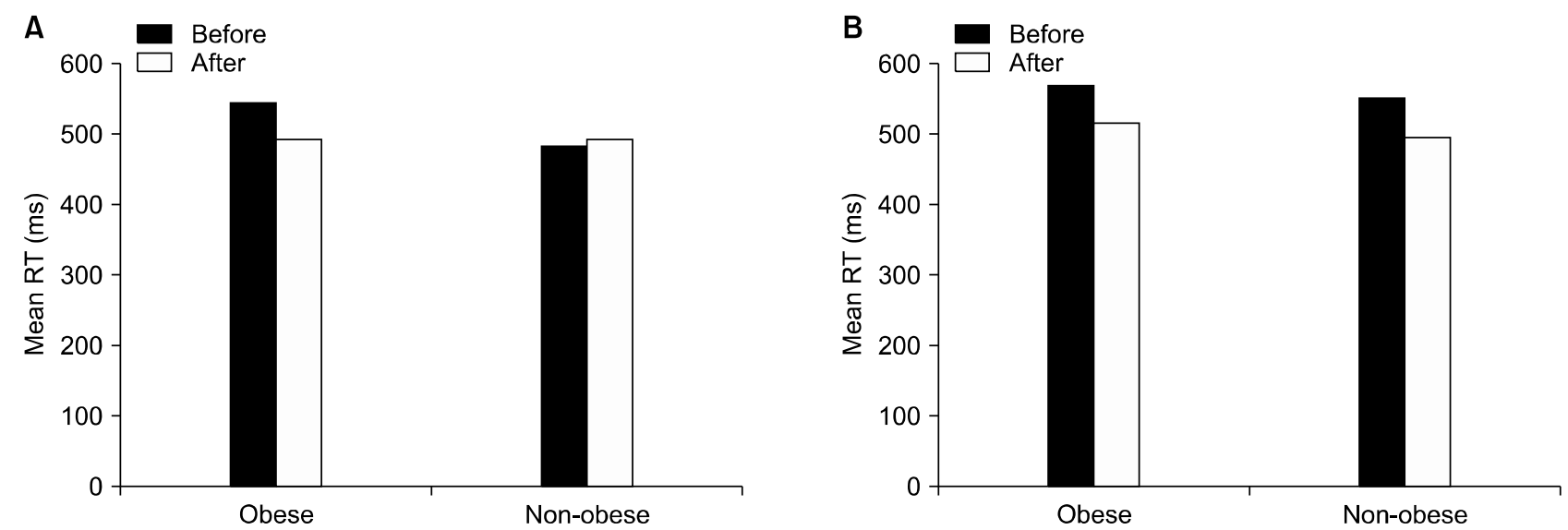

Fig. 2. Average of reaction times (RT) in auditory task (A) and visual task (B) for obese and non-obese person, before and after eating breakfast.

As shown in Figure 2, the mean of reaction times is reduced in the both auditory and visual tasks for the obese case in the fed state compared to fasting state. For the non-obese case, the reaction time is reduced in the visual task after eating breakfast, but it is increased in the audi- tory task in fed state. These observations are relatively consistent with the results obtained from SSVEP and ASSR characteristics in Figure 1.

As shown in Figure 3, we can see a clear shift towards high frequency rhythms for the both subjects after eating 
A

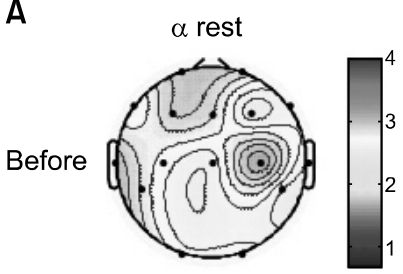

After

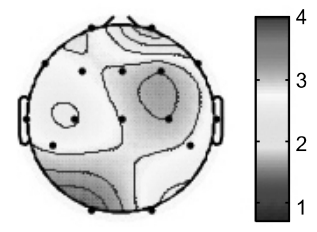

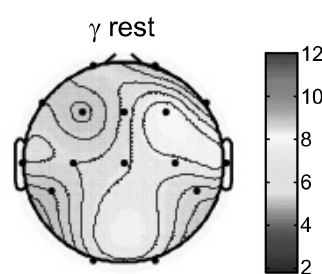

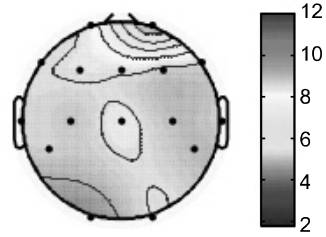

B
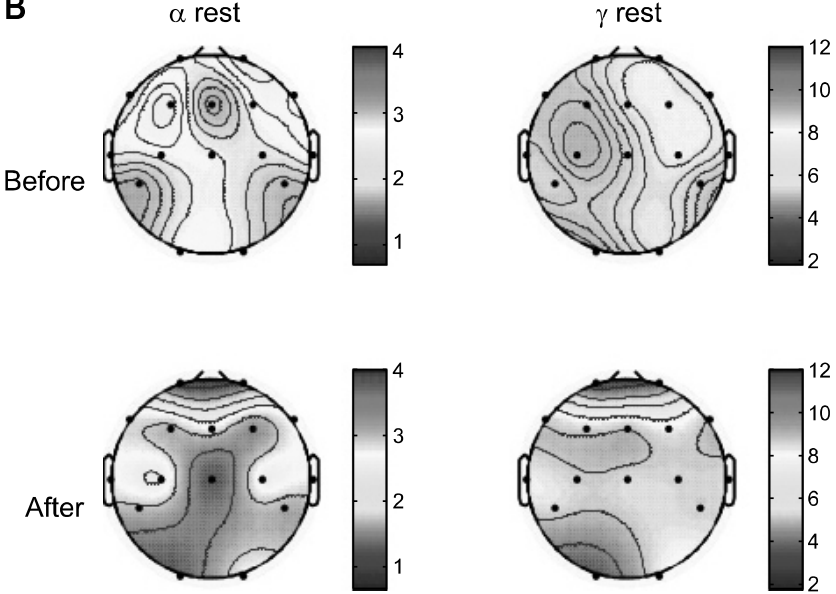
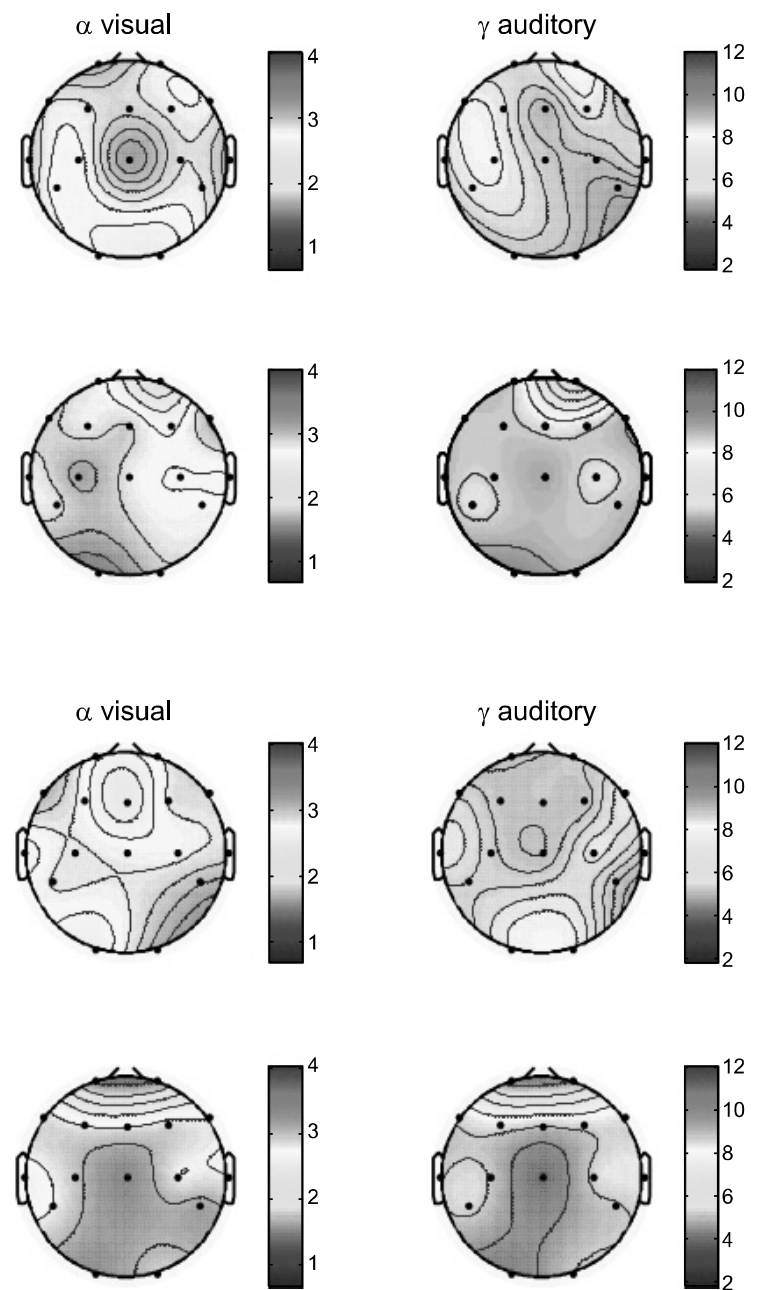

Fig. 3. Alpha activity and gamma activity in resting-state, alpha activity in visual task and gamma activity in auditory task for obese subjects (A) and non-obese subjects (B) before and after eating breakfast.

breakfast. For the both obese and non-obese cases, alpha power in fed state is higher than that in fasting state during rest and visual task. This is an interesting finding and demonstrates that less cortical resources were required to engage in the task at hand in fed state compared to fast state. In other words, suppression in alpha power corresponds to increased cognitive effort to perform the visual task in fast state. Therefore, there is an inverse relationship between cortical resources engaged in the visual task and alpha power regardless of the nutritional status of individuals, as previously stated in the literature. ${ }^{16,17)}$ Gamma power in the temporal region is increased for the obese subject after eating breakfast, but it remains almost unchanged for the non-obese subject. According to the evidence about the relationship between gamma power and reaction times in the auditory tasks, ${ }^{18,19)}$ increases in gamma activity in the temporal region result in faster reaction times for the obese subject in the fed state; whereas, unchanged gamma activity may correlate with unchanged reaction times for the non-obese person.

The results obtained from the neuro-cognitive tasks are almost consistent with steady-state potentials. Both cases performed better the Sternberg search in the fed state compared to fast state (Fig. 4). Although two cases had the similar performance in the fast state to perform Sternberg search, it can be seen clearly that the obese case had a better performance in the fed state. As shown in the Figure 5, obese case generated faster reaction times during Stroop test after eating breakfast, but non-obese case showed slightly slower reaction times during this test in the fed state.

To the best of our knowledge, the current paper is the 
A

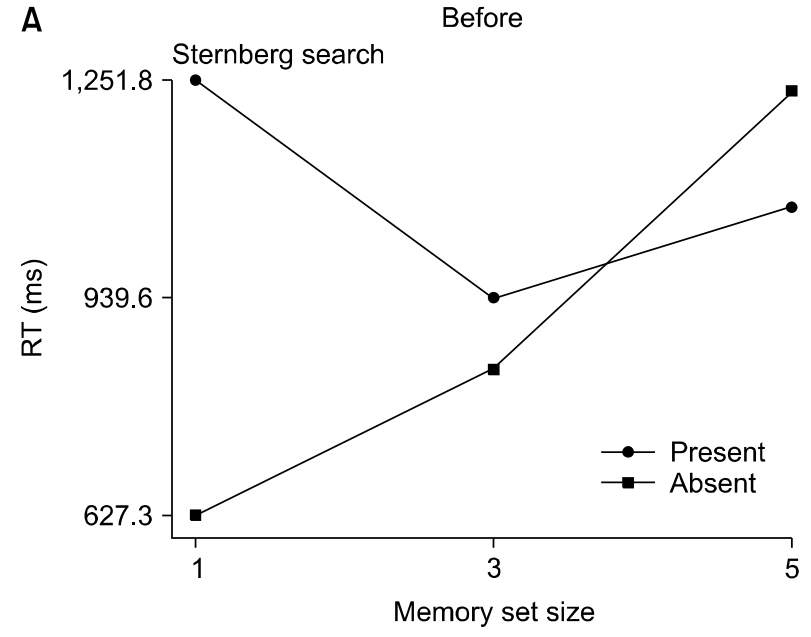

B

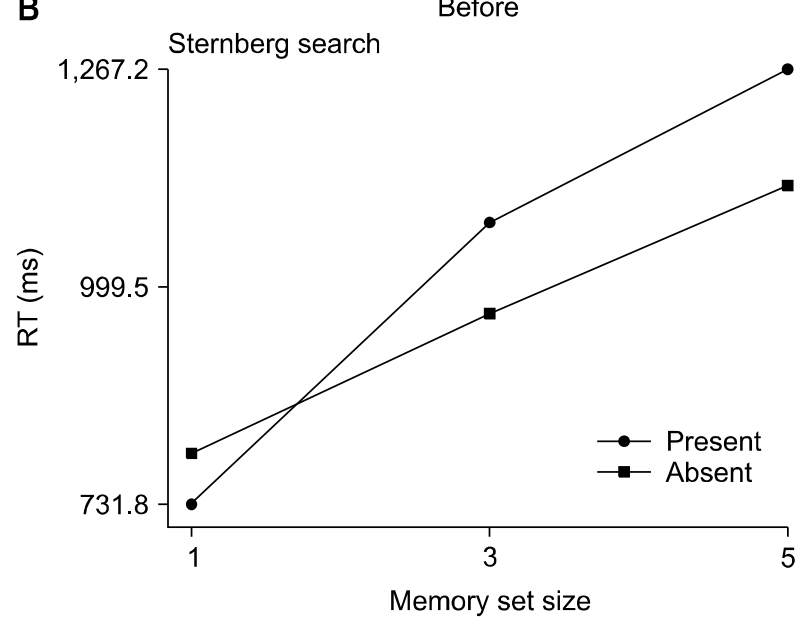

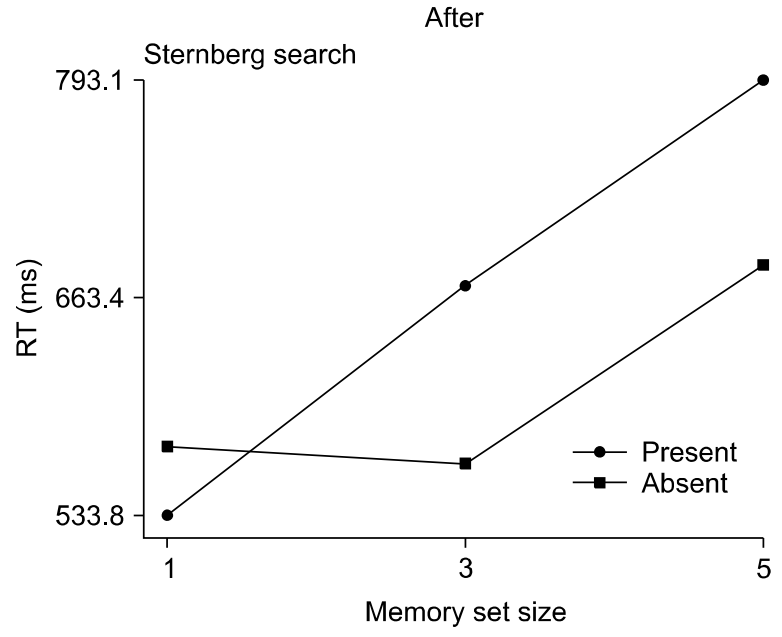

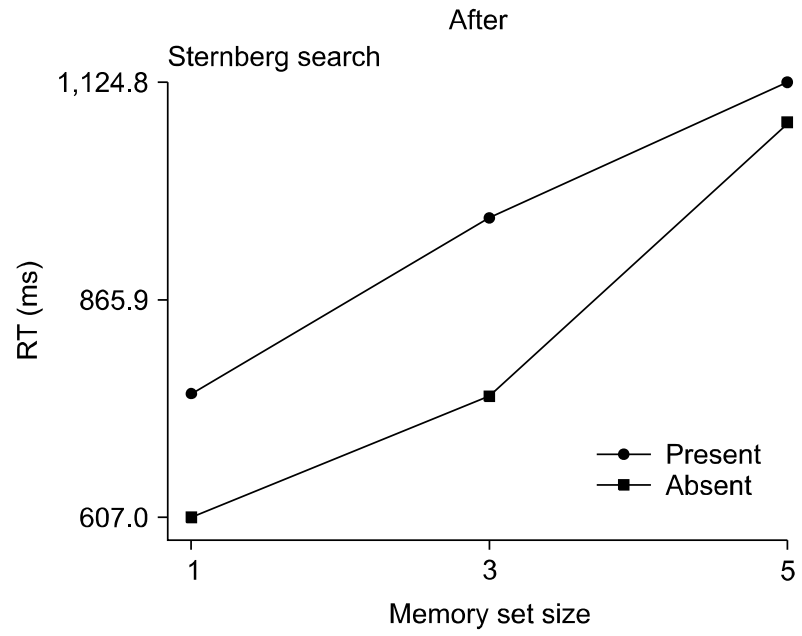

Fig. 4. Sternberg search results in obese person (A) and non-obese person (B) before and after eating breakfast. $\mathrm{RT}$, reaction time.
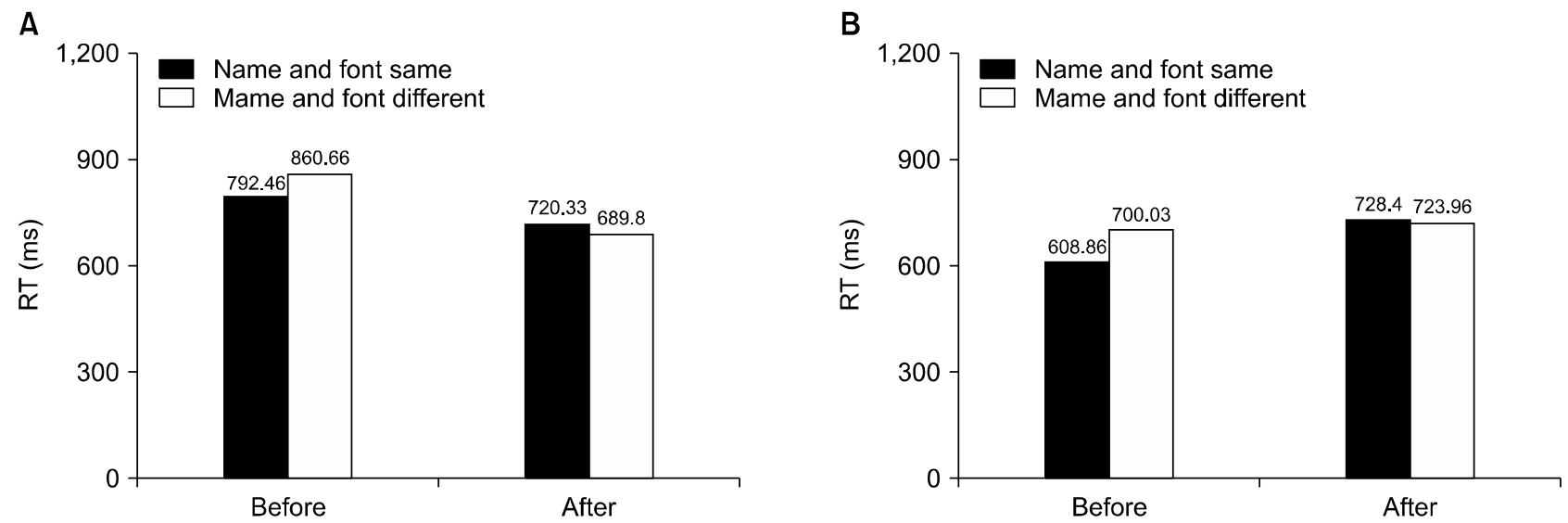

Fig. 5. Results of Stroop test in obese person (A) and non-obese person (B) before and after eating breakfast. 
first to collect neuro-cognitive ramifications of hunger and satiety in obese and non-obese cases. Breakfast may influence the neurotransmitters and micronutrient concentrations in the brain. Eating breakfast may also improve cognitive performance by increasing blood glucose levels and decreasing hunger. We think that this paper would open a new trend to answer some important questions about the neuro-cognitive implications of fasting and feeding in obese and non-obese human. It is not yet well known whether breakfast meals with different glysemic load facilitate different neuro-cognitive domains. Strengths of this study are selected cases of interest, standardized and objective tests of cognitive performance, accurately control for confounding factors, and examine mixed and iso-caloric breakfast meal.

We suggest a single component breakfast for assessing macronutrient effects on cognition and EEG, assess the other EEG measures like complexity analysis and repeat similar examinations in a large population by well-designed methodologies in the future studies to clarify ambiguous issues in this field.

\section{Acknowledgments}

We thank The Psychiatry and Psychology Research Center, Tehran University of Medical Sciences.

\section{REFERENCES}

1. Lieberman HR, Caruso CM, Niro PJ, Adam GE, Kellogg MD, Nindl BC, et al. A double-blind, placebo-controlled test of $2 d$ of calorie deprivation: effects on cognition, activity, sleep, and interstitial g/ucose concentrations. Am J Clin Nutr 2008; 88:667-676.

2. Benau EM, Orloff NC, Janke EA, Serpell L, Timko CA. A systematic review of the effects of experimental fasting on cognition. Appetite 2014;77:52-61.

3. Edefonti V, Rosato V, Parpinel M, Nebbia G, Fiorica L, Fossali $\mathrm{E}$, et al. The effect of breakfast composition and energy contribution on cognitive and academic performance: a systematic review. Am J Clin Nutr 2014;100:626-656.

4. Kim MD, Seo HJ, Yun HJ, Jung YE, Park JH, Lee Cl, et al. The relationship between cognitive decline and psychopathology in patients with schizophrenia and bipolar disorder. Clin Psychopharmacol Neurosci 2015;13:103-108.

5. Bosy-Westphal A, Later W, Hitze B, Sato T, Kossel E, Gluer $\mathrm{CC}$, et al. Accuracy of bioelectrical impedance consumer devices for measurement of body composition in comparison to whole body magnetic resonance imaging and dual X-ray absorptiometry. Obes Facts 2008; 1:319-324.

6. Mostafavi SA, Akhondzadeh S, Mohammadi MR, Eshraghian MR, Hosseini S, Chamari M, et al. The reliability and validity of the Persian version of three-factor eating questionnaire-R18 (TFEQ-R18) in overweight and obese females. Iran I Psychiatry 2017;12:100-108.

7. Mostafavi SA, Keshavarz SA, Mohammadi MR, Hosseini S, Eshraghian MR, Hosseinzadeh P, et al. Reliability and validity of the Persian version of Compulsive Eating Scale (CES) in overweight or obese women and its relationship with some body composition and dietary intake variables. Iran J Psychiatry 2016;11:250-256.

8. Mohammadi MR, Mostafavi SA, Akhondzadeh S, Keshavarz SA, Motlagh TA, Eshraghian MR, et al. Reliability and validity of the Persian version of Food Craving Questionnaire-TraitReduced (FCQ-T-r) in overweight and obese women. Tehran: Tehran University of Medical Sciences, Psychiatry and Psychology Research Center; 2017.

9. Ghassemzadeh H, Mojtabai R, Karamghadiri N, Ebrahimkhani N. Psychometric properties of a Persian-language version of the Beck Depression Inventory--Second edition: BDI-II-PERSIAN. Depress Anxiety 2005;21:185-192.

10. Parker BA, Sturm K, MacIntosh CG, Feinle C, Horowitz M, Chapman IM. Relation between food intake and visual analogue scale ratings of appetite and other sensations in healthy older and young subjects. Eur J Clin Nutr 2004;58:212-218.

11. Zarafshan H, Khaleghi A, Mohammadi MR, Moeini M, Malmir N. Electroencephalogram complexity analysis in children with attention-deficit/hyperactivity disorder during a visual cognitive task. J Clin Exp Neuropsychol 2016;38:361-369.

12. Müller-Putz GR, Scherer R, Brauneis C, Pfurtscheller G. Steady-state visual evoked potential (SSVEP)-based communication: impact of harmonic frequency components. J Neural Eng 2005;2:123-130.

13. Sternberg Search [Internet]. CogLab: The Online Cognition Lab; 2014 [cited 2017 Apr 1]. Available from: https:// coglab.cengage.com/labs/sternberg_search.shtm/.

14. Stroop JR. Studies of interference in serial verbal reactions. J Exp Psychol 1935;18:643-662.

15. Silberstein RB, Farrow M, Levy F, Pipingas A, Hay DA, Jarman FC. Functional brain electrical activity mapping in boys with attention-deficit/hyperactivity disorder. Arch Gen Psychiatry 1998; 55:1105-1112.

16. Brier MR, Ferree TC, Maguire MJ, Moore P, Spence J, Tillman $\mathrm{GD}$, et al. Frontal theta and alpha power and coherence changes are modulated by semantic complexity in Go/NoGo tasks. Int J Psychophysiol 2010;78:215-224.

17. Sauseng P, Klimesch W, Schabus M, Doppelmayr M. Fronto-parietal EEG coherence in theta and upper alpha re- 
flect central executive functions of working memory. Int I Psychophysiol 2005;57:97-103.

18. Dockstader C, Wang F, Bouffet E, Mabbott DJ. Gamma deficits as a neural signature of cognitive impairment in children treated for brain tumors. J Neurosci 2014;34:8813-8824.
19. Schadow J, Dettler N, Paramei GV, Lenz D, Fründ I, Sabel BA, et al. Impairments of Gestalt perception in the intact hemifield of hemianopic patients are reflected in gamma-band EEG activity. Neuropsychologia 2009;47:556-568. 